\title{
On the persistency of crater assignment criteria for Stromboli explosion-quakes
}

\author{
Roberto Carniel and Franco Iacop \\ Dipartimento di Georisorse e Territorio, Università di Udine, Italy
}

\begin{abstract}
On the basis of the results of previous works by our group, this paper aims to investigate the correlation between features of a kind of seismic events recorded at Stromboli (the so called explosion-quakes) and the source of such explosions, i.e. the different craters. The purpose is that of finding parameters in order to try an automatic assignment of new events to their crater of origin. These parameters must be searched for both in time and in frequency domain. Afterwards the stability in time of the discrimination is discussed. An important «side effect» of this analysis is the discovery that the percentage of summital explosions not producing seismic signals recordable by a $2 \mathrm{~s}$ seismometer is not negligible.
\end{abstract}

Key words explosion-quakes - craters

\section{Introduction}

The idea of associating some typical features of the explosion-quakes recorded by a seismic station with the activity of the different vents in the summit craters of Stromboli volcano has been around for some years, mainly suggested by the stability of the morphology (Washington, 1917) and by the persistent activity of the craters. A long term monitoring of the seismic activity is essential in order to understand the dynamics of the volcano, and therefore to estimate its actual risk (Schick and Müller, 1985).

This paper follows a previous work (Beinat et al., 1988), where correlation between seismograms, recorded during field work with portable instrumentation, and the explosive activity of the different vents had been studied. In that work, which came before the installation of our permanent seismic station on Stromboli, an interesting, although somehow heuristic, clustering procedure was tried.
The first parameter to be investigated was the delay between the onset of the seismic records and the visual observation of the explosion. The second one was the duration of the explosion, mainly based on gas emission. In spite of the considerable uncertainty due to the intrinsic difficulty of such measurements, a good separation between events belonging to different vents was achieved (fig. 1).

Additionally, a classification of waveforms was carried out, on the basis of a series of previous works (Peterschmitt and Tazieff, 1962; Falsaperla et al., 1989) which introduced the concept of morpho-types of seismograms recorded at Stromboli; additional morpho-types were introduced in another paper (Beinat et al., 1990a), in which data were analysed coming from our one-component seismic station, installed in 1989 (Beinat et al., 1990b) near the summit of the volcano.

Other features seem to be associated with the explosions at the different craters, such as the characteristic dynamics of the emission of ejecta (Ripepe et al., 1993). All these results in terms of stability suggest independent long conduits for the different craters; these con- 


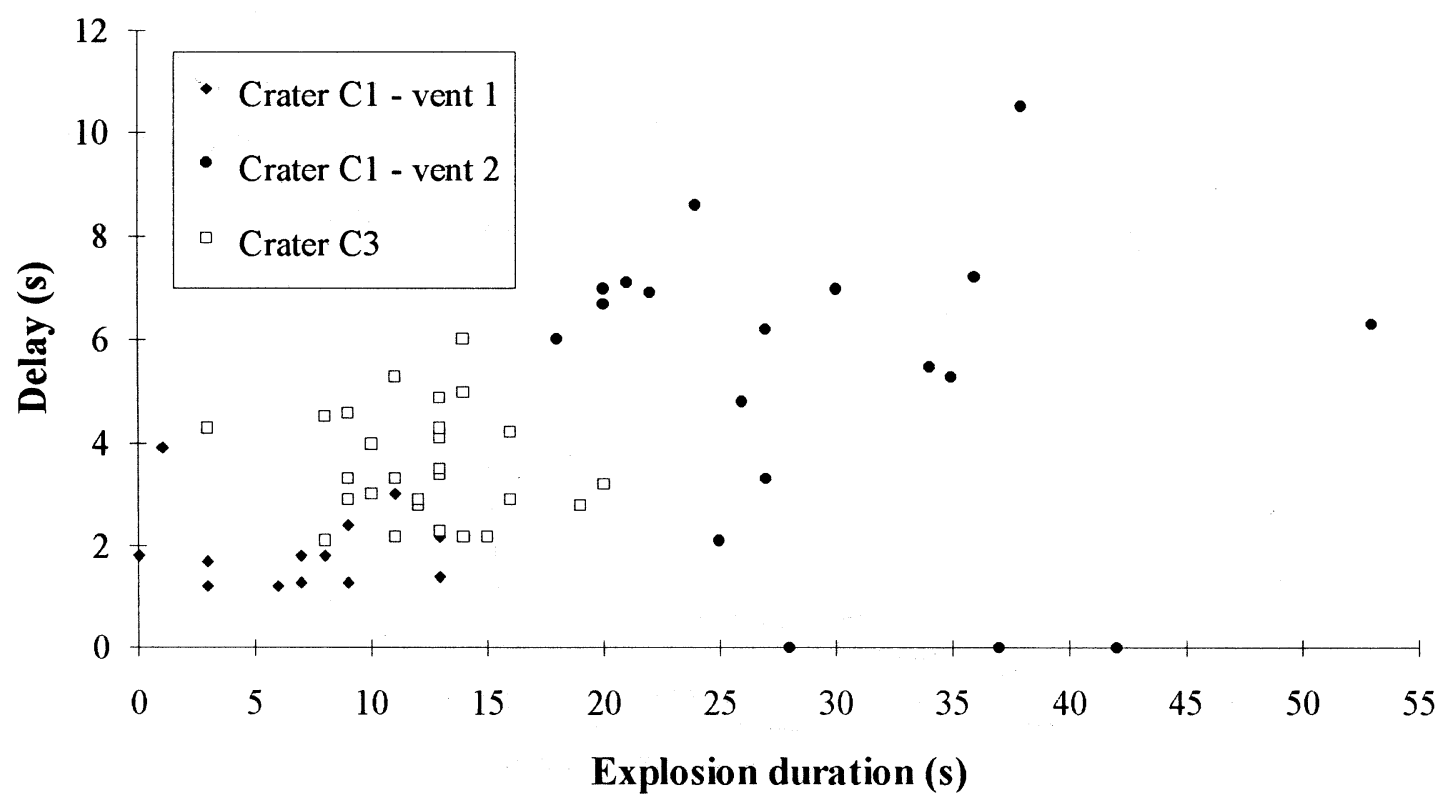

Fig. 1. Results from clustering procedure of Beinat et al. (1988).

duits are assumed to be connected quite deep in the basement (Riuscetti, 1994).

This work aims to keep on this line of research while exploiting the new features available after the upgrade of our seismic station, the most valuable of which is the presence of a complete three-component instrumentation. The data acquisition is controlled by a trigger algorithm based on a combination of amplitude-frequency thresholds and STA/LTA ratio (Beinat et al., 1994).

We correlate data recorded by our station, located at about $300 \mathrm{~m}$ from the craters, at $800 \mathrm{~m}$ a.m.s.l. (fig. 2), with visual observations made from Pizzo Sopra la Fossa, above the craters. The goal is to define a procedure for assigning the explosion-quakes to the different craters by statistical methods, using parameters describing the events, both in time and in frequency domain. If we want this procedure to be fully automatic, neither of the parameters used in Beinat et al. (1988) can be included.

The complete automatization of such a procedure of assignment of seismic events to their

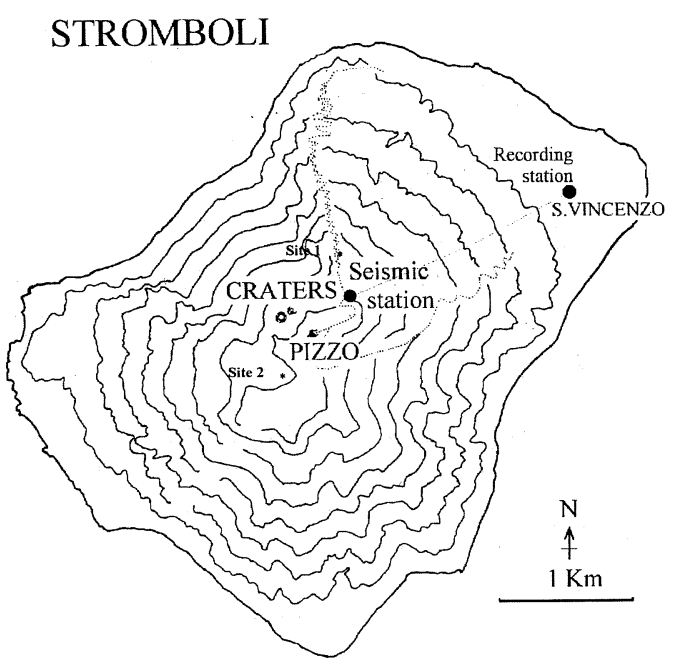

Fig. 2. Map of Stromboli with the position of the fixed seismic station and of the two sites where the MARS-88 was located. The recording station is where the digitisation and triggering of the seismic signal of the permanent station takes place. 


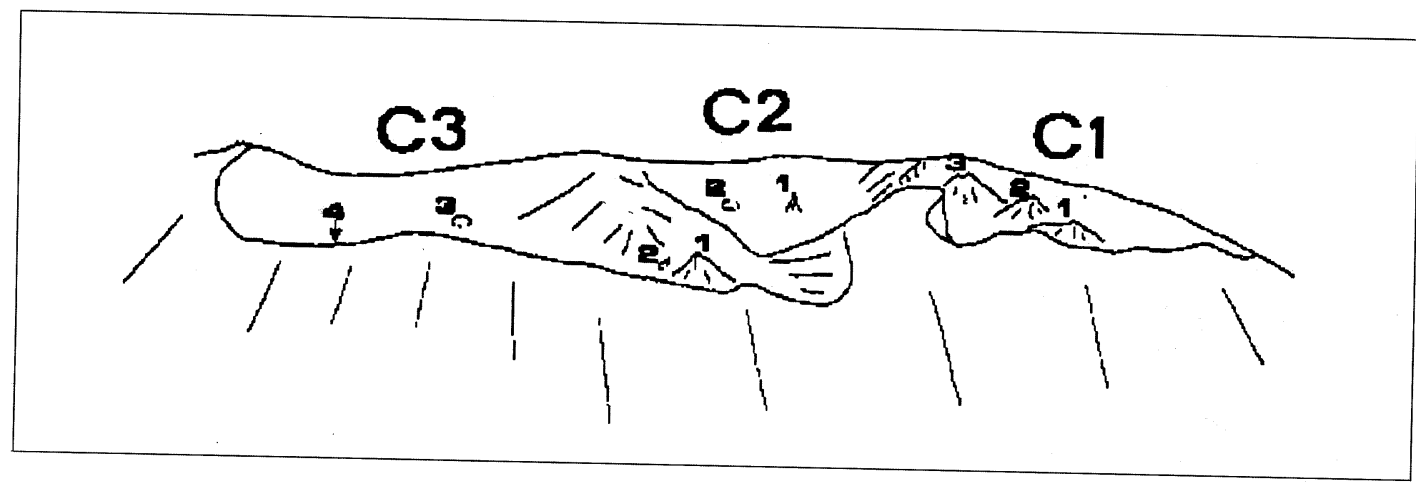

Fig. 3. Sketch of the summit craters of Stromboli as seen from Pizzo sopra la Fossa in May 1992.

crater of origin would in fact allow one to derive individual time series of the explosions at each crater. This being essential in the evaluation of the degree of interaction between the craters, it would in turn offer considerable help in the development of a model for Stromboli (Settle and McGetchin, 1980).

Although on Stromboli there are more vents than craters (fig. 3), in this paper we limit ourselves to studying the correlation between seismic events and the crater of origin. This choice is due to a number of reasons. Firstly, the activity during the initial period of observation always originated from the same vent in each crater. This seems to be confirmed by the reasonable dispersion of the values of the parameters investigated: the features of the activity could in fact be quite different for different vents of the same crater, as can be argued from the results of Beinat et al. (1988) (fig. 1). Secondly, the visual identification of a given vent in a crater, especially during the day, is not always an easy task; correlation of the explosion-quake features with the single vents may be wrongly assumed because of too many experimental errors. Finally, while there is always a slight temporal variation in the presence and in the activity of the single vents (Schick and Müller, 1985), the configuration of the three craters is a much more stable feature of the summit morphology of the volcano.

The sensors of our station are Willmore MKIII/A seismometers adjusted to a period of $2 \mathrm{~s}$, along the three components vertical, radial and tangential, the last two being referred to the direction of the craters. Very recent results, e.g. Dreier et al. (1994) and Neuberg et al. (1994), have highlighted that broadband data offer much more information than short period seismometers and therefore make the discrimination between the craters much easier. However, the use of short period data is still suggested by the lack of a permanent broadband station on the island of Stromboli as opposed to the presence of a short period station which is the only summit instrumentation which operates practically continuously on this volcano.

\section{The first data set: May 1992}

While the acquisition of seismic data is being done continuously, the visual observations, essential in order to assign the explosions to the different craters, may only be done during field campaigns. In the first stage of this work data coming from field work done on the days 12 to 18 May 1992 (Carniel and Iacop, 1993) were used.

This first data set consisted of 140 visual observations. While 11 of these could not be assigned to a crater with sufficient confidence, most of them were uniquely tagged in a way that can be relied upon: 48 were assigned to the NE crater, C1 (the nearest to the «Sciara del Fuoco», see fig. 3), while 50 were assigned to the SW crater, C3. Additionally, there were 22 observations of explosions at crater $\mathrm{C} 1$ and 
9 at crater $\mathrm{C} 3$ for which no ground motion was recorded by the seismic station. It is noteworthy however that there were no recorded events without visual counterpart.

No explosion-quakes were associated to the middle crater, $\mathrm{C} 2$; in our opinion, this could be due to the fact that the external activity of this crater in the period when the observations were made was limited to steady degassing. The corresponding seismic signal is characterized by a noteworthy component in the range below $0.5 \mathrm{~Hz}$, therefore detectable only by a broadband seismometer (Neuberg et al., 1994).

\section{Analyses in time domain}

In order to determine the parameters which best separate the explosion-quakes into disjointed subsets associated to the different craters, we first confined ourselves to the time domain.

While the maximum amplitudes of the three components did not seem to offer promising results, better results were obtained using the ratios between such maximum amplitudes. In particular, while the ratio between the vertical and the radial component did not show any separation, a much better differentiation was offered by the vertical to tangential ratio; the ratio between the maxima of the two horizontal components also offered good results. The station being quite close to the craters, the radial to tangential ratio can be interpreted as an estimate of the source direction of the seismic waves. However, path effects still affect the goodness of this estimation.

The distribution of other time domain parameters, such as the energy or some other integral parameter of the time series relative to the different components, was studied, but these values did not offer any promising way of discriminating the craters.

\section{Analyses in frequency domain}

The considerable difference in waveforms which can be noted comparing many recordings relative to explosion-quakes originated at different craters suggests that spectral data should be much more informative for tagging the events.

In fig. 4 we present an interesting example: two explosion-quakes were recorded within a minute, the first of which originated at crater $\mathrm{C} 3$, the second at crater $\mathrm{C} 1$. Here the difference in the spectral content of the two events can be inferred: the event from crater C3 seems to be characterised by a distribution which is much more concentrated in the low frequency range. This is confirmed by the analysis of the results of the Fourier transform of many other events: the ones from $\mathrm{C} 1$ present a spectrum which is spread all over the frequency range, covering frequencies exceeding $5 \mathrm{~Hz}$ with still significant peaks, while the spectra of the C3 explosion-quakes generally present most of their energy in correspondence with the low frequencies (below $3 \mathrm{~Hz}$ ). An example is presented in fig. 5 where the spectra of the tangential components of two events, the first recorded at 18:23:40 GMT of 14 May 1992 from crater C3, the second at 16:44:30 GMT of 17 May 1992 from crater C1, can be compared.

The problem is now that of representing these global differences in the features of the spectra of the two kinds of events with a single parameter. The key point is to find a measure of the «concentration» of the spectra in a given frequency range.

First of all we derive from the spectrum of each event a cumulative curve which is constructed by numerically integrating the spectrum, i.e. the value of such cumulative curve at a given frequency $f_{0}$ is simply obtained by summing up the values for all the frequencies $f<f_{0}$.

With respect to the end value of the cumulative curve, the first parameter that one can define is the percentage of the end value which is reached at a given frequency $f_{0}$. We will call this parameter the $f_{0}$ spectrum percentile. Another possibility is to go just the other way around, i.e. looking for the frequency at which a given percentage $p_{0}$ of the end value is reached. The derivation of this parameter, which we will call $p_{0}$-frequency, is shown in fig. 6 . 


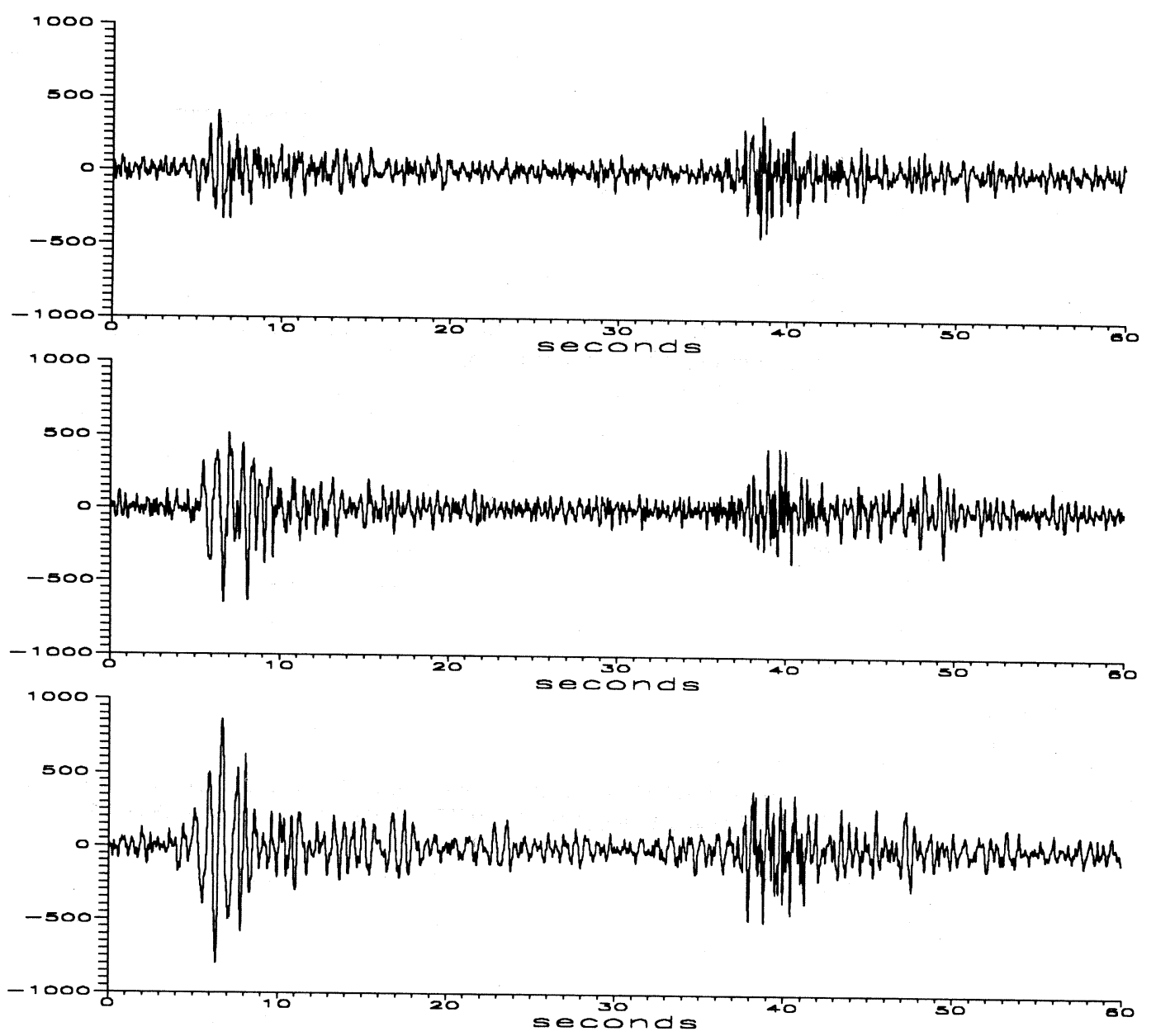

Fig. 4. Event from crater C3 followed by an event from crater C1. The record starts at 19:12:30 of 17 May 1992. The $Y$-axis is in digitisation units. The components are $Z, R, T$ from top to bottom.

\section{Time-frequency domain analysis}

We now try to combine the concentration of the spectrum distribution with the best parameters derived from the time domain analysis, i.e. the ratio of the radial to tangential and vertical to tangential component maxima.

Of course, the choice of the spectral parameter has many «degrees of freedom». In fact, we must choose first of all between the $f_{0}$ spectrum percentile and the $p_{0}$-frequency and deter- mine which is the component that we want to analyse; secondly, we must choose the value for $f_{0}$ or $p_{0}$ which best separates the events from the two craters.

We conducted many experiments assigning different values to the variables just described; heuristically, the parameters which gave the best results were the $3 \mathrm{~Hz}$ spectrum percentile and the $55 \%$ frequency. The component which seems to be the best one is the tangential one. 

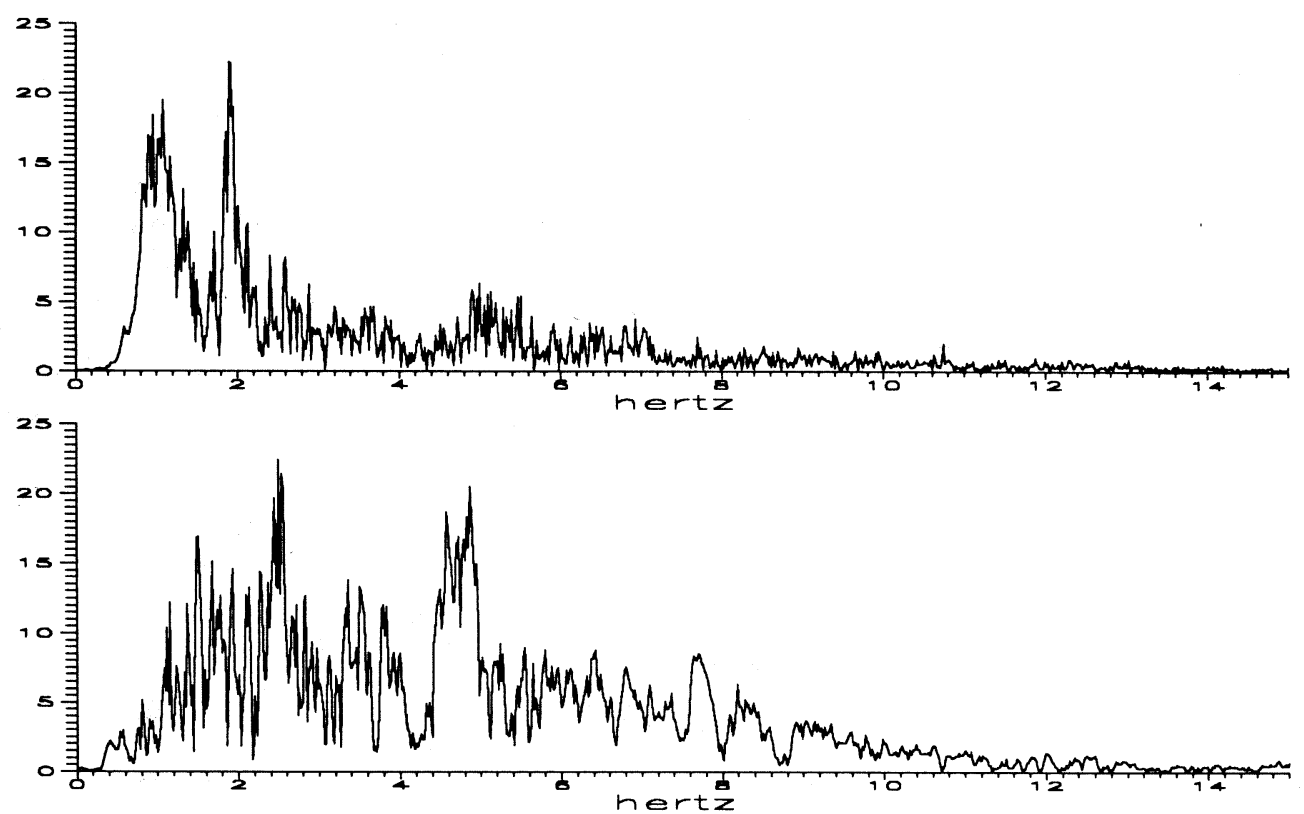

Fig. 5. Spectra of the tangential components of an event from crater C3, recorded at 18:23:40 of 14 May 1992 (top) and of one from crater C1, recorded at 16:44:30 of 17 May 1992 (bottom).

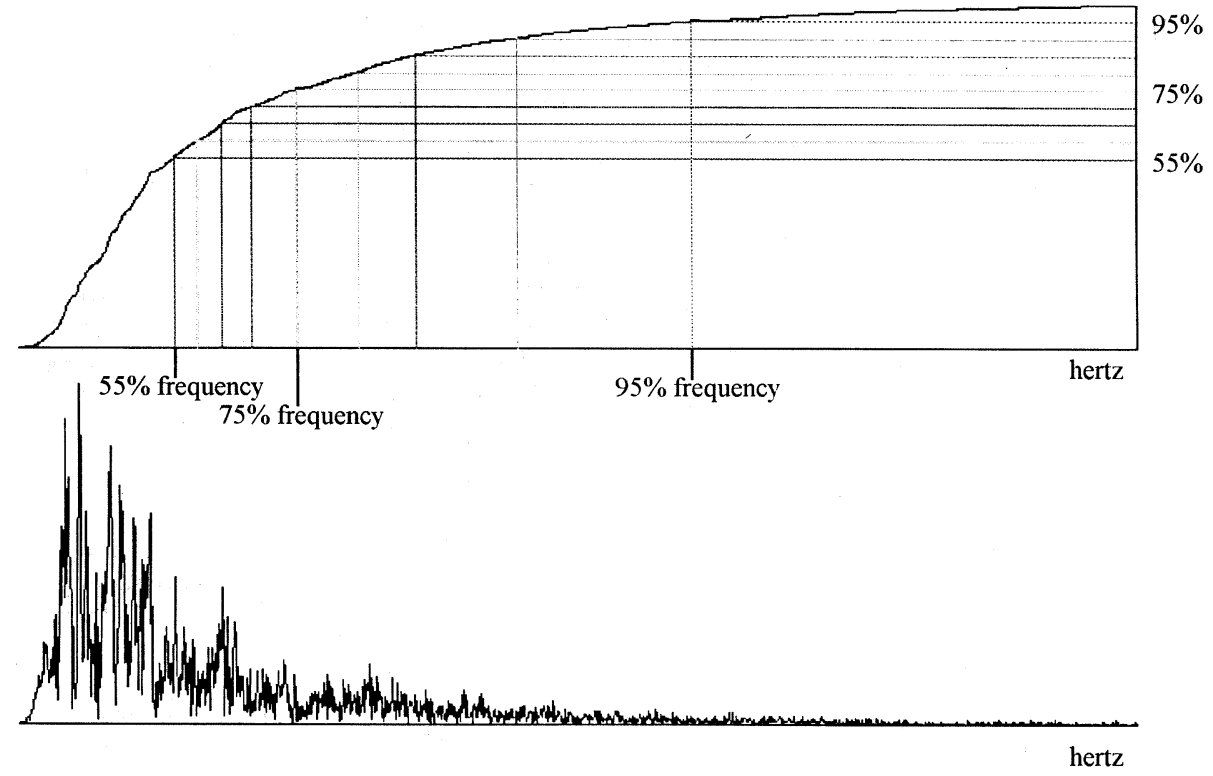

Fig. 6. Example of construction of the cumulative curve (top) from the spectrum of an event (bottom), together with the derivation of the $p_{0}$-frequency parameter for three percentages $p_{0}$ (i.e. $55 \%, 75 \%$ and $95 \%$ ) of the end value of such cumulative curve. 


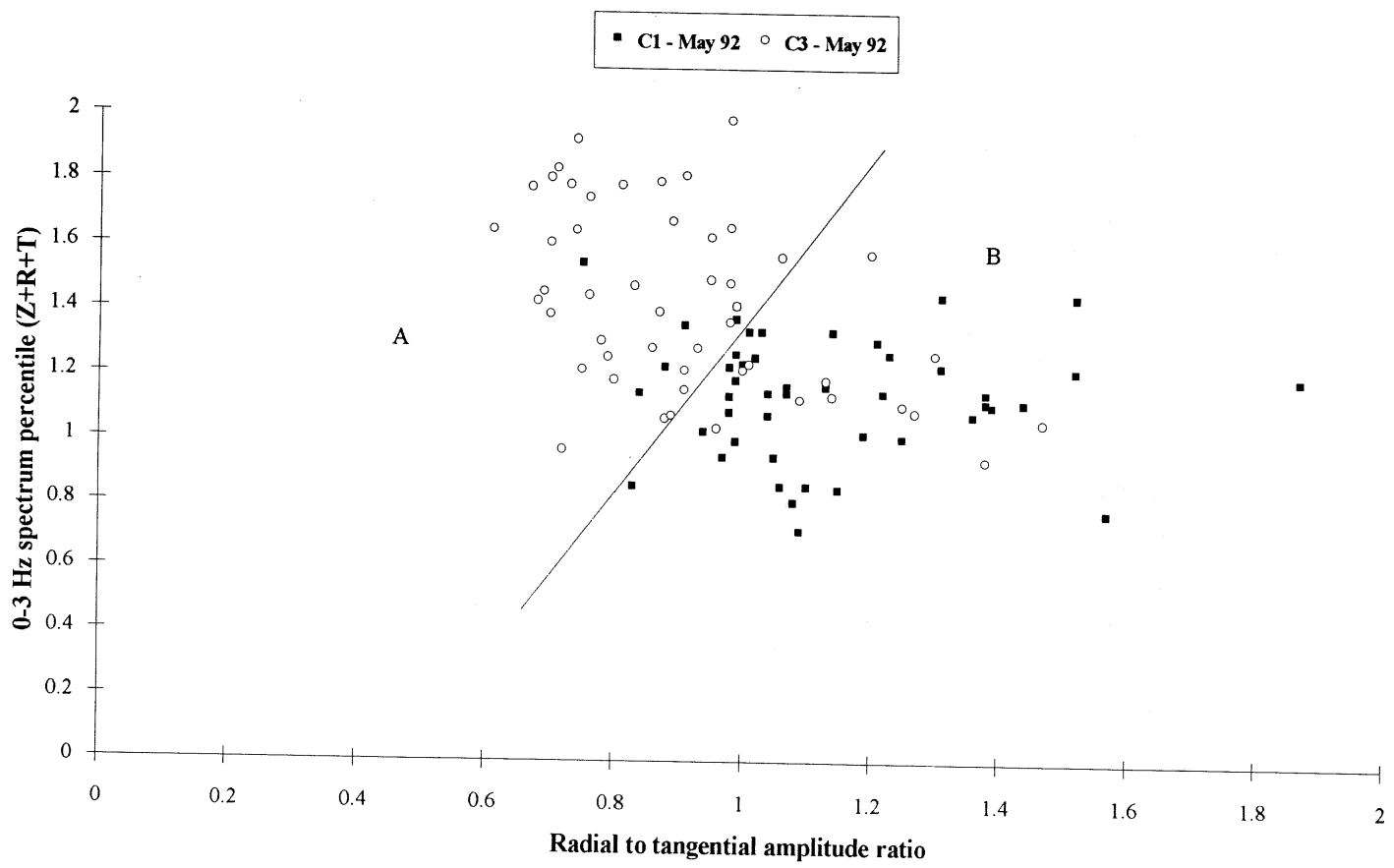

Fig. 7. Radial to tangential ratio vs. $3 \mathrm{~Hz}$ total spectrum percentile, i.e. the sum of the percentages of the total cumulative spectra reached at $3 \mathrm{~Hz}$ for the vertical, radial and tangential components of the May 1992 dataset
of explosion quakes.

In order to try to include spectral information from all three components at once, we now define a new parameter as follows: given a frequency $f_{0}$, we compute the $f_{0}$ spectrum percentile for all the components and we sum up the results. The resulting parameter, which we call total $f_{0}$ spectrum percentile, is in some way a measure of how much of the global energy of the seismic motion is concentrated in the frequency range below $f_{0}$.

Combining the total $3 \mathrm{~Hz}$ spectrum percentile with the radial to tangential maximum amplitude ratio, we obtained the best separation between the events originated at the different craters (fig. 7). The line superimposed on the scatter plot best separates the data points into two graphical areas, which we call $\mathrm{A}$ and B. The subset A contains 43 elements, 38 of which (i.e., 88\%) correspond to events originated at crater $\mathrm{C} 3$; the subset $\mathrm{B}$ contains $55 \mathrm{el}$ ements, 43 of which (i.e., 78\%) were assigned to crater $\mathrm{C} 1$. The separation obtained examining data recorded during May 1992 seems therefore to be quite good.

\section{Two other datasets: October 1992 and May 1994}

In order to examine the efficiency of the best criteria found with the May 1992 dataset, two other periods were taken into account: October 1992 and May 1994.

Due to bad weather, only 31 visual observations were taken into account in October 1992. 21 of the 27 explosions observed in $\mathrm{C} 1$ (the most active during the period of observation 11-12 October 1992) and 3 out of 4 for C3 were successfully associated to a recorded explosion-quake. The few degassing episodes observed in $\mathrm{C} 2$ were not accompanied by any 
seismic counterpart, thus confirming the May 1992 behaviour.

128 visual observations were carried out during the 22-25 May 1994 campaign, equally distributed between the craters $\mathrm{C} 1$ and $\mathrm{C} 3$; the middle crater, $\mathrm{C} 2$, was not active during the field work. The percentage of explosions accompanied by a seismic event was, on the contrary, quite different for the two craters: while for crater C3 45 out of 70 explosions were recorded by the summit station, only 11 explosions of the 58 produced in crater $\mathrm{C} 1$ were associated with an explosion-quake. It is worthwhile pointing out that the trigger algorithm (Beinat et al., 1994) was exactly the same during the three campaigns and the presence (or absence) of explosion-quakes is always confirmed by a portable seismic station equipped with a Lennartz LE-3D seismometer, located near the path leading to Pizzo Sopra la Fossa (Site 1, see fig. 2) and in Fossetta (Site 2, see fig. 2).

\section{Comparing parameters for the different datasets}

The result that one obtains by directly applying the best criteria found for the May 1992 data to the other two datasets is not very promising (fig. 8). In particular, the explosionquakes generated in crater $\mathrm{C} 3$ during May 1994 are the ones that show the worst distribution with respect to the old criteria. This means that the best criteria found for a given period are not necessarily stable in time.

In order to evaluate the performance of all the parameters the following procedure was carried out. First of all, for each period of observation the two centroids of the set of points relative to the two craters $\mathrm{C} 1$ and $\mathrm{C} 3$ were computed, the coordinates of the points being given by the different parameters; in other words, the $i$-th coordinate $P_{i, C j}$ of the centroid of crater $C j$ in a period of time is computed

- C1 - May $92 \circ$ C3-May $92 \cdot$ C1 - Oct $92 \circ$ C3-Oct $92 \backsim$ C1 - May $94 \circ$ C3-May 94

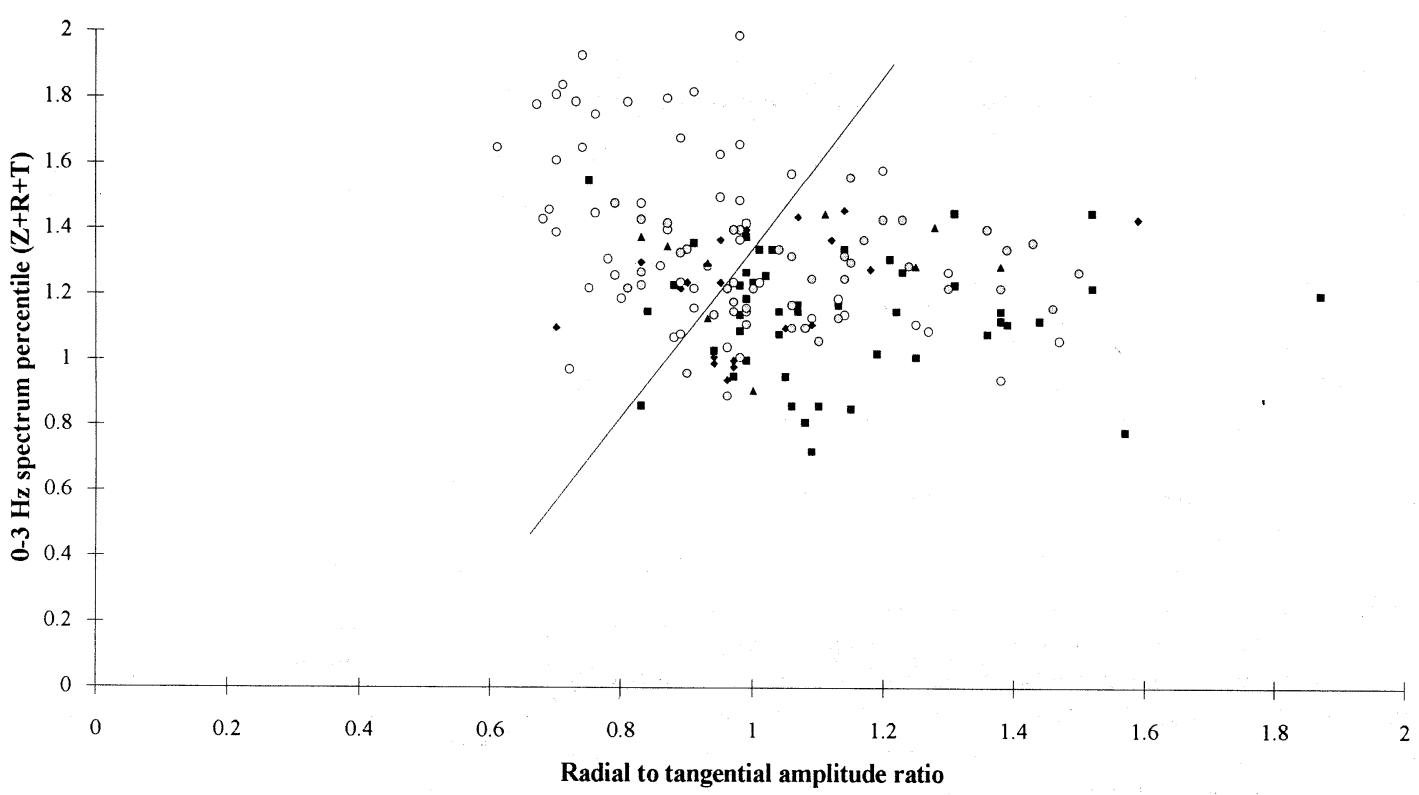

Fig. 8. Radial to tangential ratio vs. $3 \mathrm{~Hz}$ total spectrum percentile, i.e. the sum of the percentages of the total cumulative spectra reached at $3 \mathrm{~Hz}$ for the vertical, radial and tangential components of all three datasets of explosion quakes. 


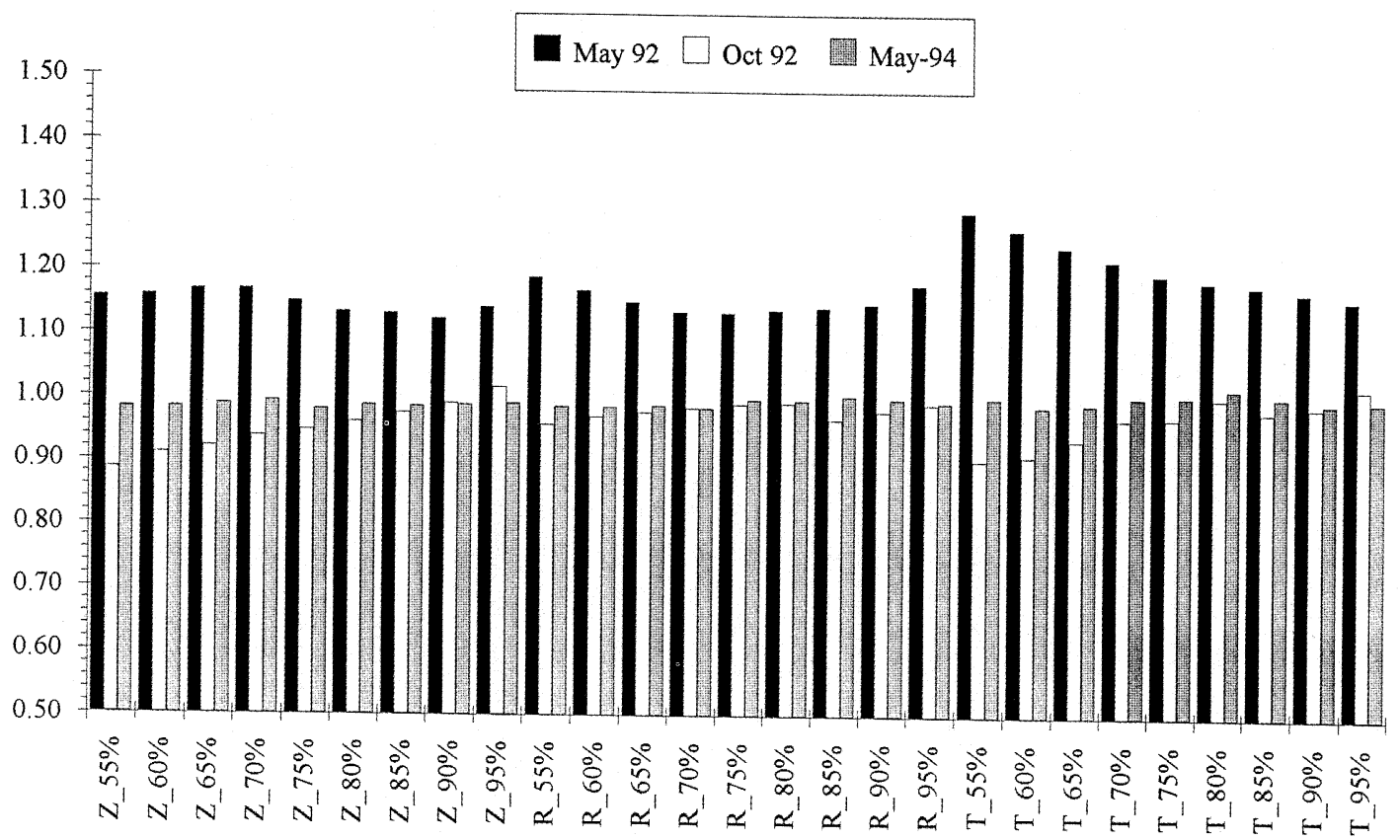

Fig. 9. Ratios of $p_{0}$-frequency coordinates of centroids (i.e. mean values) for craters $\mathrm{C} 1$ and $\mathrm{C} 3$ for the three different datasets of explosion-quakes.

simply by averaging the values of the $i$-th parameter of all the explosion-quakes associated to crater $C j$ in that period of time. For each coordinate, i.e. for each parameter considered in the analysis, the ratio $\rho_{i}=P_{i, \mathrm{C} 1} / P_{i, \mathrm{C} 3}$ was then computed and plotted (figs. 9 and 10). Of course if the $i$-th parameter does not reveal the different features of the two craters at all, the value of the ratio should be close to 1 . A first rough estimate of the ability of the parameter to discriminate between the two craters is then given by the distance of the ratio $\rho_{i}$ from the value 1 . One must in fact examine also the dispersion of the points along that coordinate.

From the plot of fig. 9, one can immediately argue that while for the May 1992 dataset all the various $p_{0}$-frequency parameters performed quite well, with a slightly better performance of the ones based on the tangential components, the same parameters perform definitely badly with the other two datasets, with values of the ratio $\rho_{i}$ always very close to 1 , especially for the May 1994 data.
The remaining parameters, presented in fig. 10, show more interesting results. First of all, according to the simple rule of the ratio, the values of the maximum amplitudes seem to be among the best parameters; this is actually not true because the values of the means for the two craters are in fact quite different but the dispersion is too high to allow the practical use of this parameter, as we had already noted while analysing the first dataset. The parameters based on the ratio between the maxima in the different components are confirmed to be quite promising; however, while for the May 1992 dataset the $Z / T$ and the $R / T$ ratios offered comparable performances, taking into account all three periods, the $Z / T$ is surely the best one to use.

In the frequency domain, it is not surprising that the $f_{0}$ spectrum percentiles that offer the best discrimination are the ones related to the low frequencies. Another confirmed feature is that the most discriminating component is the tangential one. 


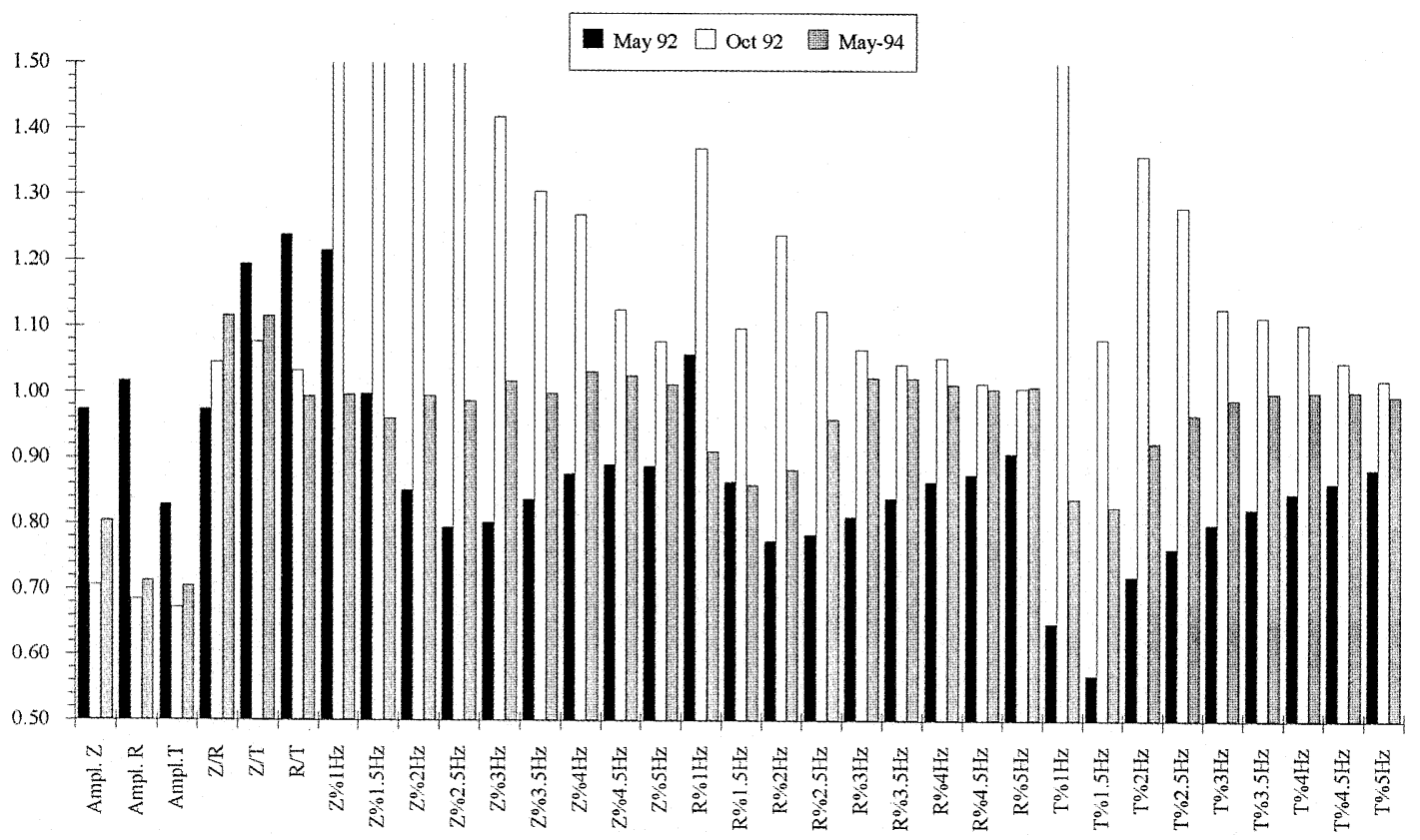

Fig. 10. Ratios of the remaining coordinates of centroids (i.e mean values) for craters $\mathrm{C} 1$ and $\mathrm{C} 3$ for the three different datasets of explosion-quakes.

The best pair of parameters to exploit both the time and frequency domain features is therefore the vertical to tangential ratio of maxima and the $1.5 \mathrm{~Hz}$ spectrum percentile. The corresponding distribution is shown in fig. 11. Even this scatter plot, however, is far from being completely satisfactory, as it does not show two sets of points sufficiently disjoint in order to define an automatic discrimination algorithm.

\section{An explanation for differences between datasets}

One could argue from fig. 11 plot that no feature remains stable across the three periods of time examined in this study. However, before drawing any conclusion in this sense it is interesting to have a direct look at the spectra. Taking for example the May 1994 explosionquakes assigned to crater $\mathrm{C} 3$, one can see that the main spectral feature highlighted for the
May 1992 data, i.e. the concentration of the spectrum in the low frequency range, is found again for many May 1994 events (as can also be seen in the plot of fig. 11). However, if we examine events which in the scatter plot do not seem to follow the criteria for being assigned to crater $\mathrm{C} 3$, we discover that the spectrum is not so different from the «typical» spectrum of May 1992. In fact, especially in the tangential component, the wide peak covering the range below $2 \mathrm{~Hz}$ is still present but it is accompanied by other significant peaks going towards the middle frequency range; this is the cause of the failure of the «concentration» criterion. But what in turn is the source for these additional peaks? We claim that these are due to volcanic tremor, which we believe due to a superposition of multiple sources (see, Julian, 1994, for a review of possible causes), including some form of resonance effects. During May 1994 the tremor level was quite high (GVN, 1994) and in our hypothesis mainly due to the activity of a small, shallow lava pond located in the 
front of crater $\mathrm{C} 3$; other authors claim that this is the only source of tremor (Ripepe and Braun, personal communication). In any case, the spectrum of the tremor presented several peaks in the same middle frequency range as the $\mathrm{C} 3$ events associated with bigger explosions. Therefore, it seems that similar spectral characteristics are masked by different tremor spectra. The difference in the spectral features of the two craters is confirmed by the analysis of the spectra recorded by the Lennartz LE-3D seismometer, although this $1 \mathrm{~s}$ instrument is less capable of highlighting the difference in the lower frequency range. Taking into account the observations described above, it is therefore necessary to go towards an analysis which takes into consideration the variations in the «spectral noise» constituted by the tremor, which shows different «stable configurations» (Carniel and Iacop, 1996).

\section{Conclusions}

In this paper an approach was made to the problem of automatically assigning explosionquakes recorded by a three-component summit seismic station to the different craters of Stromboli volcano.

Several parameters, both in the time and in the frequency domain, were defined for analyzing three datasets, relative to May 1992, October 1992 and May 1994 respectively. In the first period of time a good discrimination is achieved by means of the ratio between the maximum amplitudes of the radial and the tangential component and the total $3 \mathrm{~Hz}$ spectrum percentile - which gives a hint about the concentration of energy in the low-frequency range, possibly connected to the vibration of craters with different geometry; this demonstrates that the events can be separated with

- C1 - May $92 \circ$ C3 - May 92 • C1 - Oct $92 \circ$ C3 - Oct 92 C1 - May $94 \circ$ C3- May 94

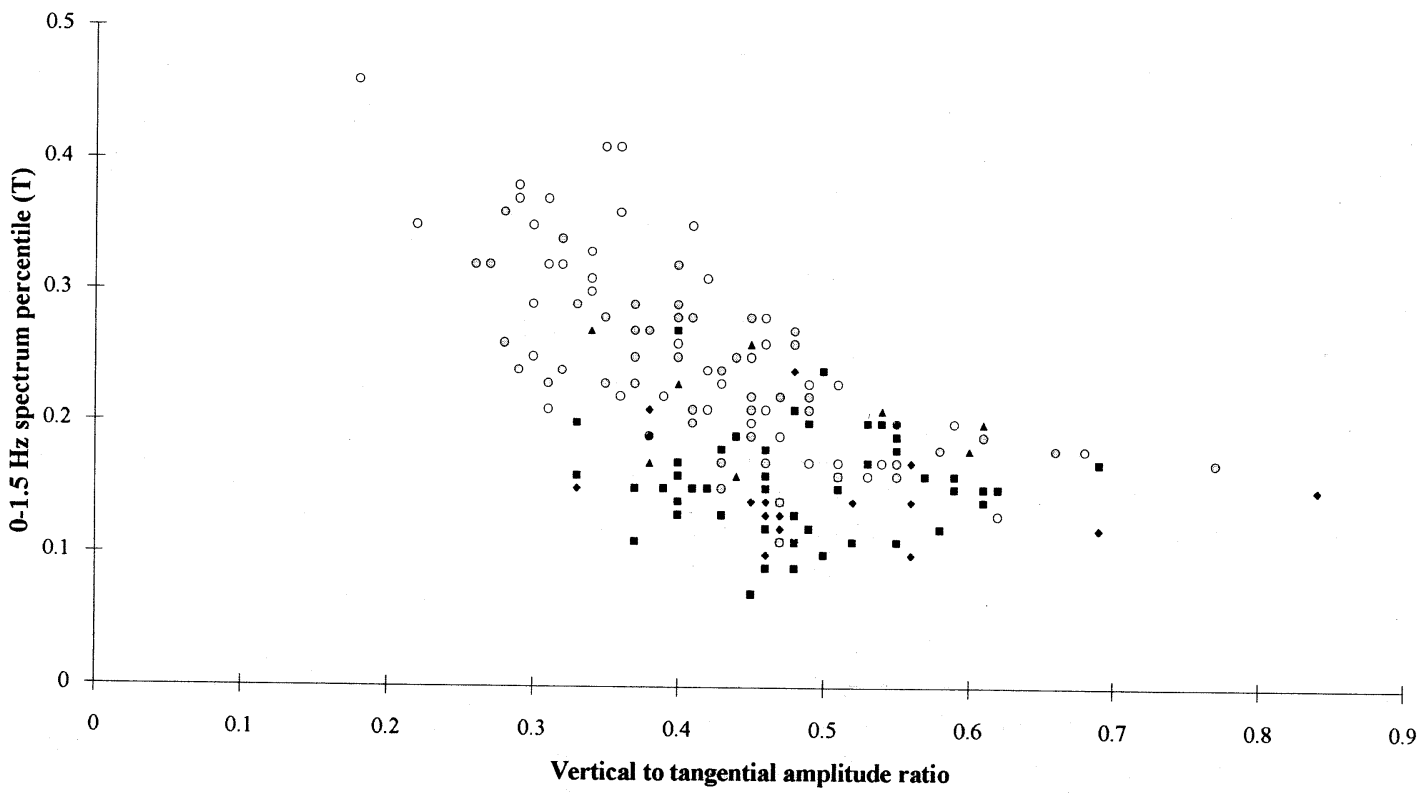

Fig. 11. Vertical to tangential ratio vs. $1.5 \mathrm{~Hz}$ tangential spectrum percentile, i.e. the percentage of the total cumulative spectra reached at $1.5 \mathrm{~Hz}$ for the tangential component of all three datasets of explosion quakes. 
sufficiently good efficiency at least for a limited amount of time.

The inclusion of the two other datasets in the analysis shows however that good separation criteria are not necessarily stable in time. Actually, the best algorithm for discriminating all the events in the three datasets makes use of the vertical to tangential amplitude ratio and of the $1.5 \mathrm{~Hz}$ spectrum percentile; however, this is still far from being completely satisfactory.

Although at first sight it seems that spectral features of the different craters do not remain the same throughout the different periods examined, at a further examination similar spectral characteristics seem to be masked by different tremor spectra. Some kind of filtering of the frequency content of the underlying tremor is therefore necessary in order to obtain better results in the discrimination.

We also think that the installation of a permanent broadband station on Stromboli, which would give new insight into the very low frequency window, could also improve the discrimination of the explosion-quakes originated at craters $\mathrm{C} 1$ and $\mathrm{C} 3$, while adding an entire new set of data, corresponding to the activity of crater $\mathrm{C} 2$ (if this crater, now quite inactive, resumes activity).

Good statistics being essential for the tuning of the separation algorithms, great help could also be obtained using long term observations of the crater area made during all good weather days by the video camera recently installed at Pizzo sopra la Fossa.

Further analyses have to be carried out in this direction. The stability of the separation algorithms with time is in fact a very important question to be answered, as the degree of stability of the explosion-quake characteristics could be useful for the development of a model of the volcano, thus contributing to the forecast of its behaviour.

With regard to this, it is also noteworthy that not all the explosions produce an appreciable seismic signal, at least according to the record of $2 \mathrm{~s}$ and $1 \mathrm{~s}$ seismometers. This behaviour, already known for the degassing activity of Crater $\mathrm{C} 2$, is quantified here also for Craters $\mathrm{C} 1$ and $\mathrm{C} 3$, for which a non trivial fraction of events lacked a seismic counterpart.
This could be a serious obstacle to obtaining reliable statistics of the explosive activity by recording the seismic activity.

\section{Acknowledgements}

The authors wish to thank M.S. Barbano and G. Salemi for their help during data acquisiton. This work was carried out with the financial support of the Gruppo Nazionale per la Vulcanologia, CNR, Italy.

\section{REFERENCES}

Beinat, A., F. Iacop, M. Riuscetti and G. Salemi (1988): Caratterizzazione statistica dell'attività sismica di Stromboli, in Atti VII Convegno Gruppo Nazionale Geofisica della Terra Solida, Roma, vol. III, 14711482.

Beinat, A., F. Iacop, M. Riuscetti and G. Salemi (1990a): First Results of Sesmic Monitoring of the Explosive Activity of Stromboli Volcano (Aeolian Islands), European Geophysical Society, XV General Assembly, Copenhagen (oral presentation).

Beinat, A., F. IAcop, M. Riuscetti and G. SAlemi (1990b): Integrated PC workstation for geophysics monitoring: a seismic application on Stromboli volcano (Aeolian islands, Italy), in Proceedings European Seismological Commission, XXII General Assembly, Barcelona, 13-19.

Beinat, A., R. CARniel and F. IACOP (1994): Seismic station of Stromboli: 3-component data acquisition system, in ESC Workshop "Dynamical Behaviour of the Strombolian Activity», Stromboli, Italy, 13-18 May 1992, Acta Vulcanol., 5, 221-222.

CARNIEL, R. and F. IACOP (1993): Waveform analysis of explosion-quakes at different vents on Stromboli, in ESC Workshop «Non-linear Analysis of Volcanic Tremor», Lanzarote (E), 24-29 May 1993.

CARNIEL, R. and F. IACOP (1996): Spectral precursors of paroxysmal phases of Stromboli, in ESC Workshop "Seismic Signals on Active Volcanoes: Possible Precursors of Volcanic Eruptions», Nicolosi, Italy, 21-25 September 1994, Annali di Geofisica, 39, 327-345 (this volume).

DREIER, R., R. WIDMER, R. SCHICK and W. ZURN (1994): Stacking of broadband seismograms of shocks at Stromboli, Acta Vulcanol., 5, 165-172.

Falsaperla, S., A. Montalto and S. Spampinato (1989): Analysis of seismic data concerning explosive sequences on Stromboli volcano in 1989, Boll. Gruppo Nazionale per la Vulcanologia CNR, 5, 249-258.

GVN (1994): Bulletin of the Global Volcanism Network, Smithsonian Institution, 19 (6), 3-4.

JULIAN, B.R. (1994): Volcanic tremor: nonlinear excitation by fluid flow, J. Geophys. Res., 99, 11859-11877. 
NeUBERG, J., R. LUCKETT, M. RIPEPE and T. BRAUN (1994): Highlights from a seismic broadband array on Stromboli volcano, Geophys. Res. Lett., 21 (9), 749-752.

Peterschmitt, E. and H. TAZIEFF (1962): Sur un noveau type de secousse volcanique enregistrée au Stromboli, C.R. Acad. Sci., 255, 1971-1973.

RiPEPE, M., M. Rossi and G. SACCOROTTI (1993): Image processing of explosive activity at Stromboli, J. Volcanol. Geotherm. Res., 54, 335-351.

RiUsCetTI, M. (1994): Seismic activity as monitored by the summit station, in ESC Workshop «Dynamical behaviour of Strombolian Activity», Stromboli, Italy,
13-18 May 1992, Acta Vulcanol., 5, 207-210.

SCHICK, R. and W. MÜLlER (1985): Volcanic activity and eruption sequences at Stromboli during 1983-1984, in Modeling of Volcanic Processes, edited by C. KING and R. SCARPA (Friedr. Vieweg and Sohn), 120-139.

Settle, M. and T. R. MCGETCHIN (1980): Statistical analysis of persistent explosive activity at Stromboli, 1971: implications for eruption prediction, J. Volcanol. Geotherm. Res., 8, 45-58.

Washington, H.S. (1917): Persistence of vents at Stromboli and its bearing on volcanic mechanism, Bull. Geol. Soc. Am., 26, 249-275. 